\title{
The Utility of Clinicopathological Correlation of Sinonasal Masses in a Tertiary Hospital
}

\author{
Abdulkarim Hasan ${ }^{1}$, Mohammed Nady², Adel A. Ibrahim³ ${ }^{3}$ Sherif Fayad ${ }^{4}$, Yasien Mohammed ${ }^{5}$, \\ Ahmad Kandil ${ }^{6}$, Nasser Omar ${ }^{7}$ \\ 1, 5, 6,7 Department of Pathology, Al-Azhar University, Cairo, Egypt. ${ }^{2}$ Department of ENT, \\ Al-Azhar University, Cairo, Egypt. ${ }^{3}$ Department of Surgical Oncology, Al-Azhar \\ University, Cairo, Egypt. ${ }^{4}$ Department of Radiology, Al-Azhar University, Cairo, Egypt.
}

\section{ABSTRACT}

\section{BACKGROUND}

Sinonasal masses are commonly seen lesions in ENT practice with multiple aetiological factors. We wanted to study the clinico-pathological features of nasal mass specimens in a tertiary hospital and evaluate the utility of routine histopathological examination of the surgically removed nasal tissue specimens.

\section{METHODS}

All the histologically examined specimens of patients clinically presenting with nasal or sinonasal lesions over a period of one year were retrospectively studied and analyzed.

\section{RESULTS}

Out of total 36 patients with masses, 21 were males (58.3\%) and $15(41.7 \%)$ were females. The age ranged from 10 to 75 years with a mean age of 31.4 years and maximum patients were in the third decade (37.5\%). The most common presenting clinical symptom was nasal obstruction that was seen in 33 patients (91.6\%). Computed tomography (CT) scan was done in $94.5 \%$ of cases prior to the surgical intervention. One fifth of the sinonasal lesions were neoplastic as per histopathology examination. The clinicopathological agreement percentage for detection of the nature of the nasal mass was $88.9 \%$. The applied interrater reliability in Cohen's kappa coefficient $(\kappa)$ showed moderate agreement (0.55).

\section{CONCLUSIONS}

The clinical similarity between the histologically different lesions is common and may lead to improper management; so, correlation of clinical, radiologic and pathologic features is of utmost importance for accurate diagnosis and the policy of discarding a surgically removed nasal lesion which clinically appears unremarkable should be totally avoided.

\section{KEY WORDS}

Nasal Polyp, Pathology, Sinonasal Carcinoma
Corresponding Author: Dr. Abdulkarim Hasan, Department of Pathology, Faculty of Medicine, Al-Azhar University, Cairo, Egypt. E-mail: doctorabdlkarim7@gmail.com

DOI: 10.14260/jemds/2021/146

How to Cite This Article:

Hasan A, Nady M, Ibrahim A, et al. The utility of clinico-pathological correlation of sinonasal masses in a tertiary hospital. J Evolution Med Dent Sci 2021;10(10):679683, DOI: $10.14260 / \mathrm{jemds} / 2021 / 146$

Submission 09-10-2020,

Peer Review 14-01-2021,

Acceptance 20-01-2021,

Published 08-03-2021.

Copyright (C) 2021 Abdulkarim Hasan et al. This is an open access article distributed under Creative Commons Attribution License [Attribution 4.0 International (CC BY 4.0)] 


\section{BACKGROUND}

Masses in nasal cavity and paranasal sinuses form a heterogeneous group of neoplastic and non-neoplastic lesions including the inflammatory polypoidal lesions. ${ }^{1}$ These neoplastic and non-neoplastic lesions are quite impossible to clinically differentiate and mostly diagnosed clinically as nasal polyp. $^{2}$ Nasal polyps are usually symptomized by nasal obstruction; however, this obstruction is very common and caused by a wide range of aetiologies. ${ }^{3}$

Various histopathologic findings ranging from nonneoplastic to sinonasal malignant tumours may mimic a simple polyp or mass. ${ }^{4}$ Computed Tomography (CT) imaging is the investigation of choice in pre-operative assessment of the nasal cavity and the paranasal sinuses and is considered the gold standard for description of the inflammatory sinus diseases resulting from obstruction. However, some drawbacks of CT are recorded in diagnosis of invasive fungal diseases and some early sinonasal polyps. ${ }^{5}$ In spite of this, some authors considered the clinical evaluation by the surgeon is sufficient to ascertain nature of the surgically removed polyp, especially, when it appears as simple nasal polyp, so the routine histopathological examination of all the nasal biopsy specimens obtained after polypectomy remains controversial. 6,7

The main aim of this study is to assess the clinicopathological features of nasal mass specimens and evaluate the importance of routine histopathological examination of the surgically removed nasal tissue specimens.

\section{METHODS}

The present study is a retrospective study performed at a tertiary care hospital in a one-year period from Jan 2019 to Jan 2020. It comprises all the biopsies of sinonasal lesions received at the histopathology laboratory. Specimens were evaluated grossly, microscopically, routine special staining and immunohistochemical study was done when required. A pathology request form usually accompanies every single case, ${ }^{8}$ all the studied cases request forms were retrieved to record the demographic data. The relative detailed clinical data was obtained from the medical files and the clinician in concern. Histological reexamination of the neoplastic lesions was done. Functional endoscopic sinus surgery (FESS), intranasal polypectomy, endoscopic medial maxillectomy and biopsy were the surgical procedures performed in the studied cases, according to the type and clinical impression of nasal mass.

\section{Statistical Analysis}

Thirty six lesions were described and correlated with the clinical and radiological features then statistically analyzed using Excel (Excel, Microsoft Corporation, Redmond, WA, USA). The interrater reliability between clinical and pathological diagnosis was statistically assessed by kappa test. It was suggested by Cohen that the kappa result be interpreted as follows: value $\leq 0$ is indicating no agreement and $0.01-0.20$ is none to slight, $0.21-0.40$ is fair, $0.41-0.60$ is moderate,
$0.61-0.80$ is substantial, and $0.81-1.00$ is almost perfect agreement. ${ }^{9}$

\section{RESULTS}

In the study population, males were 21 out of 36 patients (58.3 $\%)$ while female patients were 15 / 36 (41.7\%). The patients' age ranged from 10 to 75 years with mean age $31.4=/-16.3$. Maximum patients of sinonasal masses were found in $21-30$ years age group (37.5 \% of the patients) (Figure 1). There was no significant association between the age and gender ( $\mathrm{p}=$ 0.35).

The performed surgical procedures were as follows; 24 cases (66.7\%) were FESS, 5 cases (13.9\%) were polypectomy, 3 biopsies (8.3\%) and 4 (11.1\%) cases surgical excision, maxillectomy (Table 1).

Two patients were not advised for any radiologic investigation, but 34 patients (94.4\%) were investigated by CT scan prior to the surgical intervention (Figure 2). The radiologic-pathologic agreement was obtained in all the confirmed inflammatory cases but not all the neoplastic ones.

The most common presenting clinical symptom was nasal obstruction that was seen in 33 patients ( $91.6 \%$ ), followed by nasal discharge in 3 patients (8.4\%).

The histopathological examination revealed 29 inflammatory cases, 5 benign neoplastic lesions (Figure 3) and 2 cases of undifferentiated sinonasal carcinomas (Figure 4).

The clinical-pathological agreement percentage for detection of the nature of the nasal mass was $88.9 \%$. The applied interrater reliability Cohen's kappa coefficient $(\kappa)$ showed moderate agreement $(0.55)$.

\begin{tabular}{|ccc|}
\hline Histopathology & Number (\%) & Treatment \\
Inflammatory & $28(77.7 \%)$ & \\
Allergic nasal polyp & $-20(55.5 \%)$ & 22 FESS \& 2 biopsies \& 4 polypectomy \\
Fungal mass & $-8(22.2 \%)$ & \\
Benign neoplastic & $6(16.7 \%)$ & Surgical removal (polypectomy) \\
Haemangioma & $-1(2.8 \%)$ & 3 Endoscopic Medial maxillectomy \&2 \\
Schneiderian papilloma & $-5(13.9 \%)$ & FESS \\
Malignant mass & & Biopsy then surgical resection in \\
Sinonasal carcinoma & $2(5.6 \%)$ & collaboration with head \& neck surgical \\
stage, II & $-1(2.8 \%)$ & oncologists. \\
Sinonasal carcinoma, & $-1(2.8 \%)$ & Biopsy then radiation therapy and follow \\
stage IV & up. \\
Total & $\mathbf{3 6}(\mathbf{1 0 0} \%)$ & \\
\hline $\begin{array}{c}\text { Hable 1. Distribution of the Studied Sinonasal Masses According to the } \\
\text { Histopathology Result and Way of Management }\end{array}$ \\
\hline
\end{tabular}

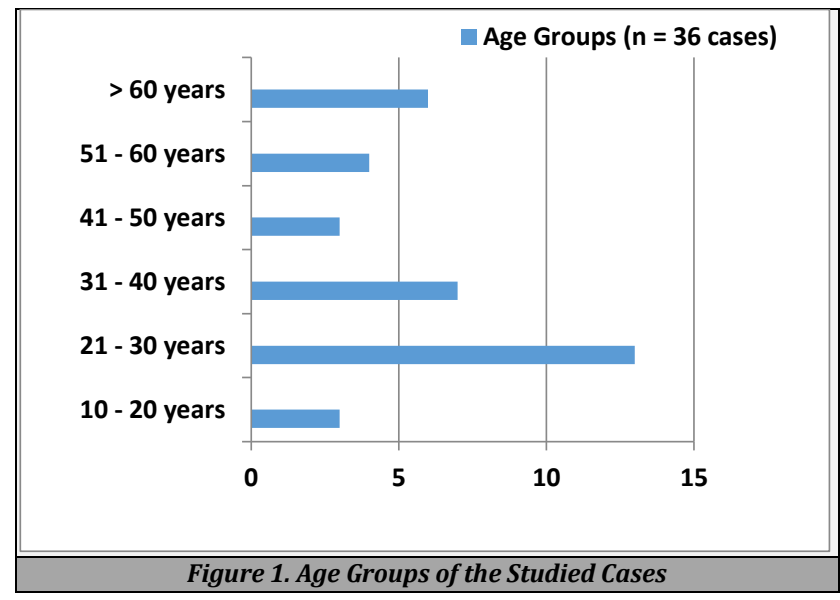



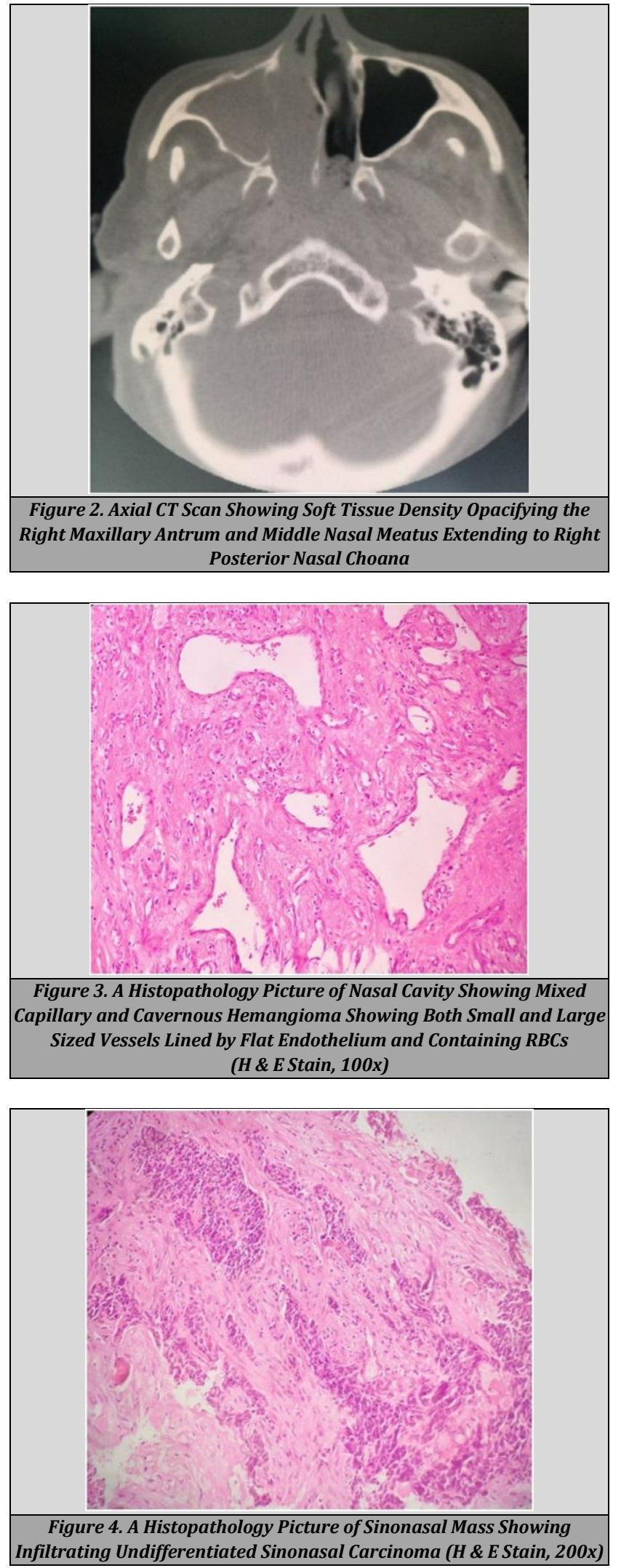

\section{DISCUSSION}

Sinonasal mass is a lesion in the nasal cavity and paranasal sinuses that may be traumatic, congenital, neoplastic (benign or malignant) or inflammatory in nature. ${ }^{10,11}$ It is a commonly encountered lesion in ENT outpatient department. The incidence of these nasal masses is often stated between $1 \%$ and $4 \%$ of the population. ${ }^{12}$

Maximum patients (37.5\%) in this study were found in 21 - 30 years age group with mean age of 31.4 in all patients which is consistent with the study done by Hakami et al. in Saudi Arabia in 2019 reporting mean age 31.7 years, ${ }^{13}$ and quite less than the mean age in the study done by Bist et al. in India in $2012^{4}$ that was 39.4 years. In one study, the mean age of nasal mass presentation was 22.5 years for the non-neoplastic lesions, 26.7 years for benign lesions, and 35.4 years for malignant lesions. ${ }^{14}$

This study shows male predominance over females (ratio 1.4:1), in agreement with Garg and Mathur study ${ }^{15}$ (ratio 1.98:1), Raj Jyothi et al. study ${ }^{16}$ with ratio 1.2:1 and Majumdar et al. study ${ }^{17}$ with ratio 1.57:1. Sharma et al. in 201718 also recorded male predominance but with higher ratio (3.1:1). This male predominance can be explained by the smoking habits, dust exposure, more infection and outdoor work in males as compared to females. $15,16,17$

Most patients usually present with complaints of nasal obstruction with one or more of other symptoms including nasal discharge, disturbances of smell, epistaxis, facial swelling, headache, oral symptoms, orbital symptoms and ear symptoms. ${ }^{19,20,21}$

Polyps are a common cause of this blockage of the nasal cavity which are often bilateral and multiple. ${ }^{12}$ More than 90 $\%$ of patients in this study were complaining of nasal obstruction and the rest of patients complained of nasal discharge. A thorough assessment of clinical features, CT scan findings and histopathological evaluation assist in better management ${ }^{12}$ Clinically, it is impossible to determine what pathology lies underneath. ${ }^{21}$ Many different histopathological tumour types are found in nasal cavity and paranasal sinuses. ${ }^{22}$

Intranasal tumours may present as typical proliferative lesions with tissue destruction or may mimic inflammatory conditions in the early stages showing polyps on endoscopy. ${ }^{23}$ Although nasal endoscopy with biopsy procedure remains the gold standard for reaching diagnosis but the presence of marked soft tissue involvement with bony destruction on CT scan raises the suspicion of a possible neoplastic lesion. ${ }^{22}$ At the same time, malignant lesions should be distinguished from non-malignant neoplastic lesions. ${ }^{23.24}$ The non-malignant neoplastic lesions in this study were haemangioma and Schneiderian papilloma (3 exophytic papillomas and 2 inverted papillomas), whereas the malignant lesions were 2 nasopharyngeal carcinomas in age group of more than 50 years. One of them was not clinically suspected. Histopathological examination is crucial and cost-effective diagnostic tool for the detection of the nature of various masses in nasal cavity or any other location. $22,24,25,26$

The variation in clinical and histopathological examination is high in this study which was noted in 4 patients $(11.1 \%)$ however, the previous similar studies showed different histopathologic findings from their clinical diagnoses in 0.3 - 4 $\%$ of cases.4,27,28 In our case, there was an inflammatory polyp which was diagnosed clinically as a fungal mass but could not find any evidence of fungal infection during the microscopic examination that might be missed during processing, but still the percentage of different diagnoses raises the importance of routine histopathology examination for all the removed 
sinonasal lesions including the inflammatory and benign looking excised lesions.

Classification of the benign lesions is essential for proper management and follow up. In spite of the benign nature of some neoplastic lesions some clinically behave as potentially notorious pathology if not excised adequately and followed adequately.21,29 Inverted papilloma is a benign lesion associated with a small proportion of sinonasal squamous cell carcinoma in addition to high propensity of recurrence and local aggressiveness. ${ }^{30}$ Haemangioma is not commonly seen in nasal cavity, though if it appears, is found attached to the nasal septum and predominantly capillary type. ${ }^{21}$ Mixed capillary and cavernous type haemangioma is seen among the cases in this study. Malignant tumours of sinonasal tract are rare. The most-common subtype of epithelial tumours is sinonasal squamous cell carcinoma, which predominantly occurs in the maxillary sinus and nasal cavity. ${ }^{31}$ Relatively little is known about incidence and prognosis of maxillary lesions in the Middle East. Surgical excision is the most common modality of treatment in most of the non-neoplastic and benign neoplastic sinonasal lesions with wide excision, chemotherapy and radiotherapy in malignant lesions with follow up. ${ }^{1}$

\section{CONCLUSIONS}

Sinonasal mass lesions are a common problem in ENT practice causing diagnostic and therapeutic dilemma. The clinical similarity between the histologically different lesions is common and may lead to improper management; so, correlation of clinical, radiologic and pathologic features is of utmost importance for accurate diagnosis and discarding a surgically removed nasal lesion is totally not recommended.

Data sharing statement provided by the authors is available with the full text of this article at jemds.com.

Financial or other competing interests: None.

Disclosure forms provided by the authors are available with the full text of this article at jemds.com.

\section{REFERENCES}

[1] Swami SV, Birare SD, Dalve KT, et al. Clinicopathological study of non-neoplastic and neoplastic sinonasal lesions. Journal of Diagnostic Pathology and Oncology 2017;2(2):40-3.

[2] Dasgupta A, Ghosh RN, Mukherjee C. Nasal polypshistopathologic spectrum. Indian J Otolaryngol Head Neck Surg 1997;49(1):32-7.

[3] Almutairi A, Alghunaim M, Alsalim AA, et al. Satisfaction of patients who underwent a septorhinoplasty with or without allergic rhinitis: a cross-sectional study at a tertiary hospital. Medical Science 2020;24(101):429-34.

[4] Duvoisin B, Landry M, Chapuis L, et al. Low-dose CT and inflammatory disease of the paranasal sinuses. Neuroradiology 1991;33(5):403-6.

[5] Khadim MT, Ahmed S, Akhtar F, et al. Clinicopathological characteristics of nasal polyps with chronic sinusitis. Journal of Bahria University Medical and Dental College Karachi, Pakistan 2016;6(3):178-81.
[6] Prior AJ, Calderon MA, Lavelle RJ, et al. Nasal biopsy: indications, techniques and complications. Respir Med 1995;89(3):161-9.

[7] Hasan A, Nafie K, Abbadi O. Histopathology laboratory paperwork as a potential risk of COVID-19 transmission among laboratory personnel. Infection Prevention in Practice 2020;2(4):100081.

[8] McHugh ML. Interrater reliability: the kappa statistic. Biochem Med (Zagreb) 2012;22(3):276-82.

[9] Agarwal P, Panigrahi R. Sinonasal mass-a recent study of its clinicopathological profile. Indian J Surg Oncol 2017;8(2):123-7.

[10] Belli S, Yildirim M, Eroglu S, et al. Single - sided sinonasal mass: a retrospective study. North Clin Istanb 2018;5(2):139-43.

[11] Rawat DS, Chadha V, Grover M, et al. Clinico-pathological profile and management of sino-nasal masses: a prospective study. Indian J Otolaryngol Head Neck Surg 2013;65(2):388-93.

[12] Hakami K, Basurrah M, Alshehri AM, et al. Clinical, histopathological and radiological features of unilateral nasal mass in Saudi Arabia: a retrospective study. Saudi Journal for Health Sciences 2020;9(1):45-9.

[13] Bist SS, Varshney S, Baunthiyal V, et al. Clinicopathological profile of sinonasal masses: an experience in tertiary care hospital of Uttarakhand. Natl J Maxillofac Surg 2012;3(2):180-6.

[14] Khan N, Zafar U, Afroz N, et al. Masses of nasal cavity, paranasal sinuses and nasopharynx: a clinicopathological study. Indian J Otolaryngol Head Neck Surg 2006;58(3):259-63.

[15] Garg D, Mathur K. Clinico-pathological study of space occupying lesions of nasal cavity, paranasal sinuses and nasopharynx. J Clin Diagn Res 2014;8(11):FC04-7.

[16] Raj JA, Sharmila PS, Shrivastava M, et al. Morphological spectrum of lesions in the sinonasal region. Journal of Evolution of Medical and Dental Sciences 2013;2(37):7175-87.

[17] Majumdar AB, Sarker G, Biswas D, et al. Clinicopathological study of sino-nasal masses. National Journal of Otorhinolaryngology Head and Neck Surg 2014;2(11):1.

[18] Sharma A, Garg LN, Baisakhiya N. Clinico-pathological and radiological assessment of benign nasal masses. International Journal of Otorhinolaryngology and Head and Neck Surgery 2017;3(4):1087-94.

[19] Humayun AH, Huq AZ, Ahmed ST, et al. Clinicopathological study of sinonasal masses. Bangladesh Journal of Otorhinolaryngology 2010;16(1):15-22.

[20] Santhosh U, Rao MSV. Inferior turbinectomy for nasal obstruction - study of 219 cases. J Evol Med Dent Sci 2013;2(9):1371-5.

[21] Ibrahim AH, Metib NJ, Kehiosh HJ. Clinicopathological profile of patients with unilateral sinonasal masses in Karbala. Karbala Journal of Medicine 2016;9(1):2408-16.

[22] Kumar A, Sood N, Gautam R, et al. Histopathological analysis of lesion of nasal cavity, paranasal sinus and nasopharynx-a clinical study. Journal of Advanced Medical and Dental Sciences Research 2017;5(11):90-2. 
[23] Euteneuer S, Sudhoff H, Bernal-Sprekelsen M, et al. Malignomas of the nasal cavity and the paranasal sinuses: clinical characteristics, therapy and prognosis of different tumour types. Laryngorhinootologie 2004;83(1):33-9.

[24] Hasan A, Deyab A, Monazea K, et al. Clinico-pathological assessment of surgically removed abdominal wall endometriomas following cesarean section. Ann Med Surg. 2021;62: 219-24.

[25] Hasan A, Nafie K, Aldossary MY, et al. Unexpected histopathology results following routine examination of cholecystectomy specimens: How big and how significant? Ann Med Surg 2020;60:425-30.

[26] Hasan A, Nafie K, El-Sayed S, et al. Enterobius vermicularis in appendectomy specimens; clinicopathological assessment: cross sectional study. Ann Med Surg 2020;60:168-72.
[27] Kale SU, Mohite U, Rowlands D, et al. Clinical and histopathological correlation of nasal polyps: are there any surprises? Clin Otolaryngol Allied Sci 2001;26(4):321-3.

[28] Garavello W, Gaini RM. Histopathology of routine nasal polypectomy specimens: a review of 2,147 cases. The Laryngoscope 2005;115(10):1866-8.

[29] Hasan A, Nafie K, Monazea K, et al. A rare case of recurrent eccrine poroma underlying gluteal abscess. Int J Surg Case Rep 2020;75:29-31.

[30] Pitak-Arnnop P, Bertolini J, Dhanuthai K, et al. Intracranial extension of schneiderian inverted papilloma: a case report and literature review. Ger Med Sci 2012;10:Doc12.

[31] Llorente JL, López F, Suárez C, et al. Sinonasal carcinoma: clinical, pathological, genetic and therapeutic advances. Nat Rev Clin Oncol 2014;11(8):460-72. 